\title{
New records of Orchidaceae from Bali, Indonesia
}

\author{
D. Sulistiarini, D. Arifiani \& Y. Santika \\ Herbarium Bogoriense, Research Center for Biology, \\ Indonesian Institute of Sciences, Jl. Raya Jakarta-Bogor KM 46, \\ Cibinong, Bogor, Indonesia \\ dsulistiarini@yahoo.com
}

\begin{abstract}
During plant inventory work in the forested areas of Mt Mesehe and Mt Merbuk, Jembrana district, Bali, twenty orchid species were collected. Among the species collected, five were confirmed as new records for Bali. The five newly recorded species are Calanthe zollingeri Rchb.f., Plocoglottis plicata (Roxb.) Ormerod, Podochilus serpyllifolius (Blume) Lindl., Styloglossum speciosum (Blume) T.Yukawa \& P.J.Cribb, and Vanilla albida Blume. Descriptions of taxa and images are provided.
\end{abstract}

Keywords. Calanthe, Mt Mesehe, new records, orchids, Plocoglottis, Podochilus, Styloglossum, Vanilla

\section{Introduction}

Bali is famous for its beautiful landscapes and beaches, for which the island has become the number one tourist destination in Indonesia. In contrast, the plant diversity of Bali is not well known. There are few floristic publications about Bali, including little information on the orchid species of the island.

Recent classifications of the Orchidaceae recognise five subfamilies, namely Apostasioideae, Vanilloideae, Cypripedioideae, Orchidoideae and Epidendroideae, with about 27,800 species in total (Stevens, 2015). Schuiteman et al. (2014) have reported that in Malesia there are about 6000 species of which 200 are found in the Lesser Sunda Islands (Bali, Nusa Tenggara Barat and Nusa Tenggara Timur). For Bali alone, 146 species of orchids have been recorded (Girmansyah et al., 2013). Comber (1990) suggested that Java had 731 species, while Sumatra had a higher number of 1118 species (Comber, 2001). However, more recently, Schuiteman et al. (2014) have increased the number of orchid species known from Java and Sumatra to 769 and 1126 species, respectively. Therefore, it is to be expected that more species are to be found in Bali than those recorded by Girmansyah et al. (2013).

Most herbarium specimens deposited in the Herbarium Bogoriense (BO) were collected in the central and eastern parts of Bali, while fewer specimens are from the western parts of the island. Therefore, an attempt was made recently to explore plant diversity in the western parts, especially in Jembrana district in the forested areas of Mt Merbuk (1386 m asl.) and Mt Mesehe (1300 m asl.). These are the two highest mountains in Jembrana district. 
Jembrana district, which covers $841.8 \mathrm{~km}^{2}$ or $14.93 \%$ of Bali, is situated at $8^{\circ} 03^{\prime} 40^{\prime \prime}-8^{\circ} 50^{\prime} 48^{\prime \prime} \mathrm{S}$ and $114^{\circ} 25^{\prime} 53^{\prime \prime}-114^{\circ} 42^{\prime} 40^{\prime \prime} \mathrm{E}$. The topography is gently rolling country to hilly, with a fine-grained soil texture in which the grey-brown alluvial and regosol types are dominant. Jembrana district has two seasons, i.e., the rainy and dry season. The heavy rainfall occurs from December to March and the dry season usually starts from April or May. The average rainfall in 2012 was 1663 mm/year with 112 rainy days per year on average (Anonymous, 2014).

In the Mt Mesehe area, the Yeh Mesehe waterfall (at c. $980 \mathrm{~m}$ asl.) is an important water source for the villages in the region. In the Mt Merbuk area, big rivers, such as Tukad Aya, Titis and Ijo Gading, are important for maintaining forest conditions. In Jembrana, $39.17 \%$ of the land area consists of protected forests which in turn preserves the water catchment. The forests in the Mt Mesehe area are in a good condition from $350 \mathrm{~m}$ asl upwards with cultivated areas are only below this altitude. The villagers grow cacao, coffee, banana and coconut. From $900 \mathrm{~m}$ asl upwards the forest is very moist and foggy, the litter is rather thick to about $10 \mathrm{~cm}$, and available trails to explore the area are very steep. Cyathea sp., Rhododendron sp. and Vaccinium sp. are commonly found at this elevation. On Mt Merbuk wild palms are common at around $500 \mathrm{~m}$ asl, whereas at 600 to $760 \mathrm{~m}$ altitude Dipterocarpus sp., Podocarpus sp., Fagraea sp. and Pavetta sp. are common. Along the Titis river, there are, amongst others, Nephrolepis biserrata (Sw.) Schott, Asplenium nidus L., Dryopteris sp., Selaginella sp., bryophytes, Begonia sp. and some orchids. The rattans Daemonorops melanochaetes Blume and Calamus javensis Blume, along with climbers such as Freycinetia javanica Blume and members of the Vitaceae are also commonly found.

\section{Methods}

A plant inventory was conducted in the Jembrana district in the vicinity of Mt Mesehe in May 2013 and in the Mt Merbuk forest area in April 2014. The collection of orchids followed the methodology of Van Balgooy (1987). The collections were photographed when flowering and then dried as herbarium specimens. Some spirit samples of flowers were also prepared using a mix of glycerine $40 \%$, alcohol $96 \%$ and distilled water at a ratio of 1:70:29 (Rugayah et al., 2004). Sterile specimens were also collected for a permanent record of all orchid species in Bali. All measurements in the descriptions are based on dried specimens.

Five of the orchid species found are new records as they are not included in the recently published checklist of the Flora of Bali (Girmansyah et al., 2013) and their occurrence in Bali is not noted in eMonocot (2015), Schuiteman et al. (2014), Comber (1990, 2001), Seidenfaden \& Wood (1992) and Wood et al. (1993). Many orchid species are reported to be distributed in the Lesser Sunda Islands (LSI) without further elaboration on which specific island(s) they can be found. In this paper we do not include those species as new records. 


\section{Results and Discussion}

The exploration of Mt Mesehe and Mt Merbuk has resulted in many new orchid specimens of 20 species (Table 1) for Herbarium Bogoriense (BO). Previously only about sixty orchid species have been recorded from Bali but among the 20 species newly collected we were able to confirm that five species are new records. Four of these species belong to the subfamily Epidendroideae, i.e. Calanthe zollingeri Rchb.f., Plocoglottis plicata (Roxb.) Ormerod and Podochilus serpyllifolius (Blume) Lindl., Styloglossum speciosum (Blume) T.Yukawa \& P.J.Cribb. The remaining species is Vanilla albida Blume belonging to the subfamily Vanilloideae. Epidendroideae includes $78 \%$ of the species of Orchidaceae and is characterised by the presence of incumbent anthers which bend forward during column elongation (Stevens, 2015; Freudenstein $\&$ Chase, 2014). In Indonesia, Styloglossum speciosum and Plocoglottis plicata were previously known from Java, Maluku, Sulawesi, Kalimantan and Sumatra. Calanthe zollingeri, Podochilus serpyllifolius and Vanilla albida were previously known from Java, Kalimantan and Sumatra. These species are relatively common, yet they have not been recorded from Bali before.

Apart from the five newly recorded species, there are another eleven that have previously been recorded from the Lesser Sunda Islands but further study is needed to ascertain whether those records included Bali.

\section{Description of taxa}

Calanthe zollingeri Rchb.f., Bonplandia 5: 38 (1857); Comber, Orchids Java: 93 (1990). - TYPE: Java, Banyuwangi, Leg. ign. 2858 (holo W, n.v.). (Fig. 1)

Pseudobulbs close together, bearing 4-5 leaves. Leaves $23-40 \mathrm{~cm}$ long, 8-9 cm wide, ovate, acuminate, petiole 10-12 cm long. Inflorescences $40-60 \mathrm{~cm}$ tall, rachis c. $6 \mathrm{~cm}$ long, bearing 10-20 flowers, not all open at one time. Flowers white, about $2.5 \mathrm{~cm}$ wide; sepals and petals about $1 \mathrm{~cm}$ long, oblong; lip white and then turning yellow to orange, unlobed, circular, deeply split, outer margin incurved.

Distribution. Peninsular Malaysia, Sumatra, Java, Bali, Borneo.

Habitat. Lowland forest along river, also in primary forest.

Specimens examined. INDONESIA. Bali: Jembrana district: Mendoyo subdistrict, Batu Agung village, Panca Seming, forest surrounding Mt Sekar, $550 \mathrm{~m}$ asl., 17 May 2013, N. Utami 2032 (BO); Jembrana subdistrict, Dauh Waru village, Munduk Waru, forest surrounding Mt Merbuk, 520 m asl., 23 Apr 2014, Y. Santika 749 (BO); Jembrana subdistrict, Batu Agung village, Palungan Batu, Tuntuna, along Tukad Aya river, forest surrounding Mt Merbuk, $200 \mathrm{~m}$ asl., 23 Apr 2014, S. Sunarti 755 (BO). 
Plocoglottis plicata (Roxb.) Ormerod in Comber, Orchids Sumatra: 293 (2001). - Limodorum plicatum Roxb., Fl. Ind. ed. 3: 465 (1832). - Plocoglottis acuminata Blume, Ann. Mus. Bot. Lugduno-Batavum 1: 46 (1849). - TYPE: Java, Blume s.n. (L, n.v.). (Fig. 2)

Pseudobulbs well-spaced along rhizome, 4-6 cm long, $0.2-0.5 \mathrm{~cm}$ wide, cylindrical, usually covered by leaf sheath. Rhizome creeping, branched, internodes $1.5-2.5 \mathrm{~cm}$ long, $0.3-0.4 \mathrm{~cm}$ thick. Leaf 1, ovate, 20-25 cm long, 7-8.5 cm wide, acuminate, petiole 4-7 cm long. Inflorescences from base of young pseudobulb, 30-40 cm long, bearing several flowers. Flowers opening widely, yellow with red markings, $3 \mathrm{~cm}$ wide; sepals and petals narrowly lanceolate, acute; lip in outline almost square.

Distribution. Sumatra, Java, Bali, Borneo, Moluccas, Sulawesi, Philippines.

Habitat. Lowland forest along river; primary forest, sometimes growing on rocks.

Specimens examined. INDONESIA. Bali: Jembrana district: Mendoyo subdistrict, Poh Santen village, Pasatan, forest surrounding Mt Mesehe, $400 \mathrm{~m}$ asl., 18 May 2013, D. Arifiani 1365 (BO); Mendoyo subdistrict, Batu Agung village, Panca Seming, Yeh Mesehe protected forest, 860 m asl., 17 May 2013, Y. Santika 548 (BO); Jembrana subdistrict, Batu Agung village, Palungan Batu, Pangkung Palir, along Tukad Aya river, forest surrounding Mt Merbuk, $280 \mathrm{~m}$ asl., 23 Apr 2014. D. Arifiani 1416 (BO).

Podochilus serpyllifolius (Blume) Lindl., J. Proc. Linn. Soc., Bot. 3: 37 (1858); Comber, Orchids Java: 202 (1990). - Cryptoglottis serpyllifolia Blume, Bijdr. Fl. Ned. Ind.: 296 (1825). - TYPE: ?Indonesia, T. Lobb 155 (AMES, n.v.). (Fig. 3)

Pseudobulbs absent. Stems hard, creeping, hanging, branching. Leaves very small, in two rows along the stem, about $1 \mathrm{~mm}$ apart, ovate, 3-4 mm long, c. $1 \mathrm{~mm}$ wide, sessile, acute. Inflorescences usually terminal, bearing up to 4 flowers. Flowers not opening widely, about $4.5 \mathrm{~mm}$ long and $1.5 \mathrm{~mm}$ wide, white with a pink-purple spot in the middle of the petal and lip.

Distribution. Sumatra, Java, Bali, Borneo.

Habitat. Primary forest, growing in moss on rocks.

Specimen examined. INDONESIA. Bali: Jembrana district: Mendoyo subdistrict, Batu Agung village, Panca Seming, Yeh Mesehe protected forest, $935 \mathrm{~m}$ asl., 17 May 2013, Y. Santika 570 (BO). 


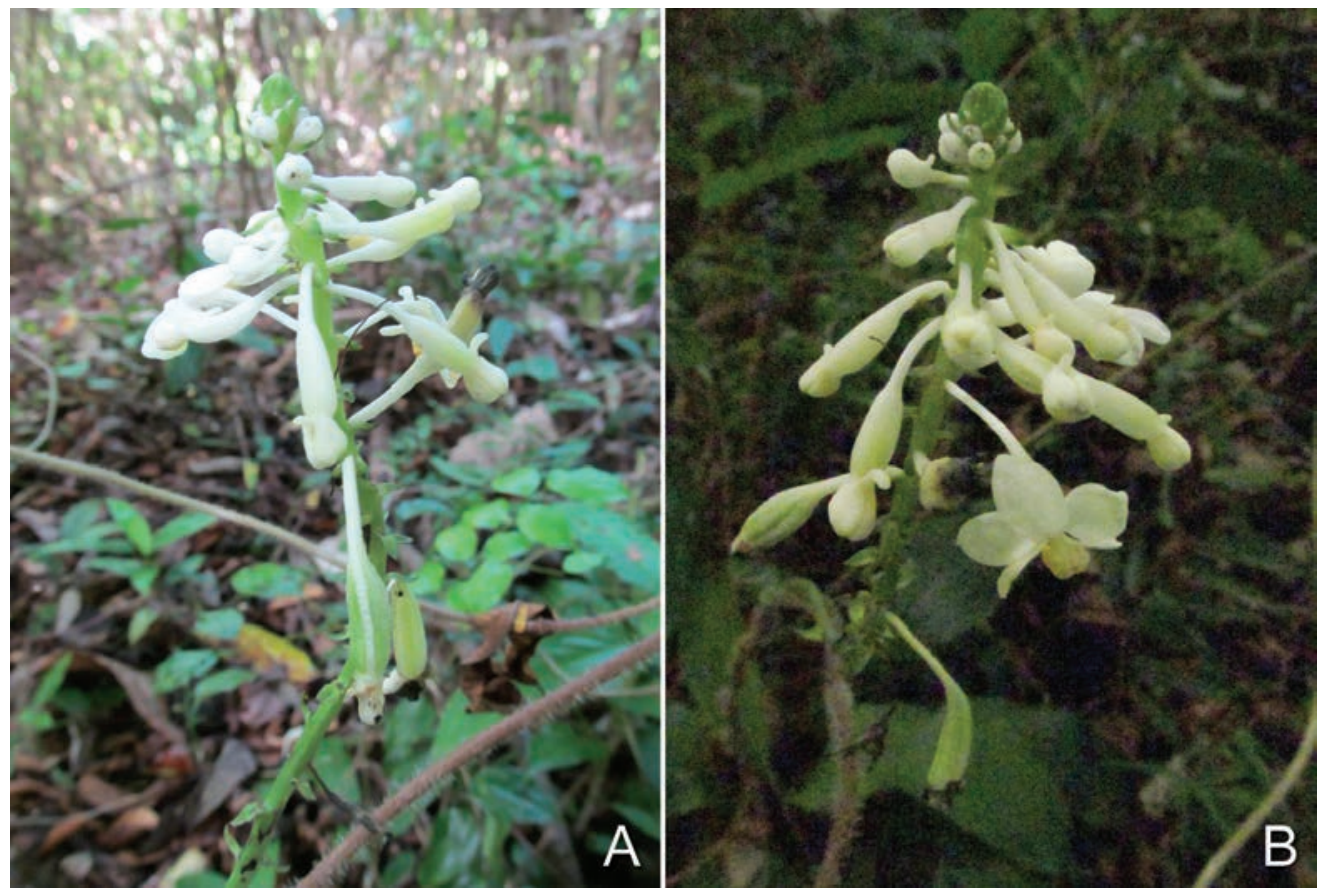

Fig. 1. A-B. Calanthe zollingeri Rchb.f. (Photos: Yessi Santika)
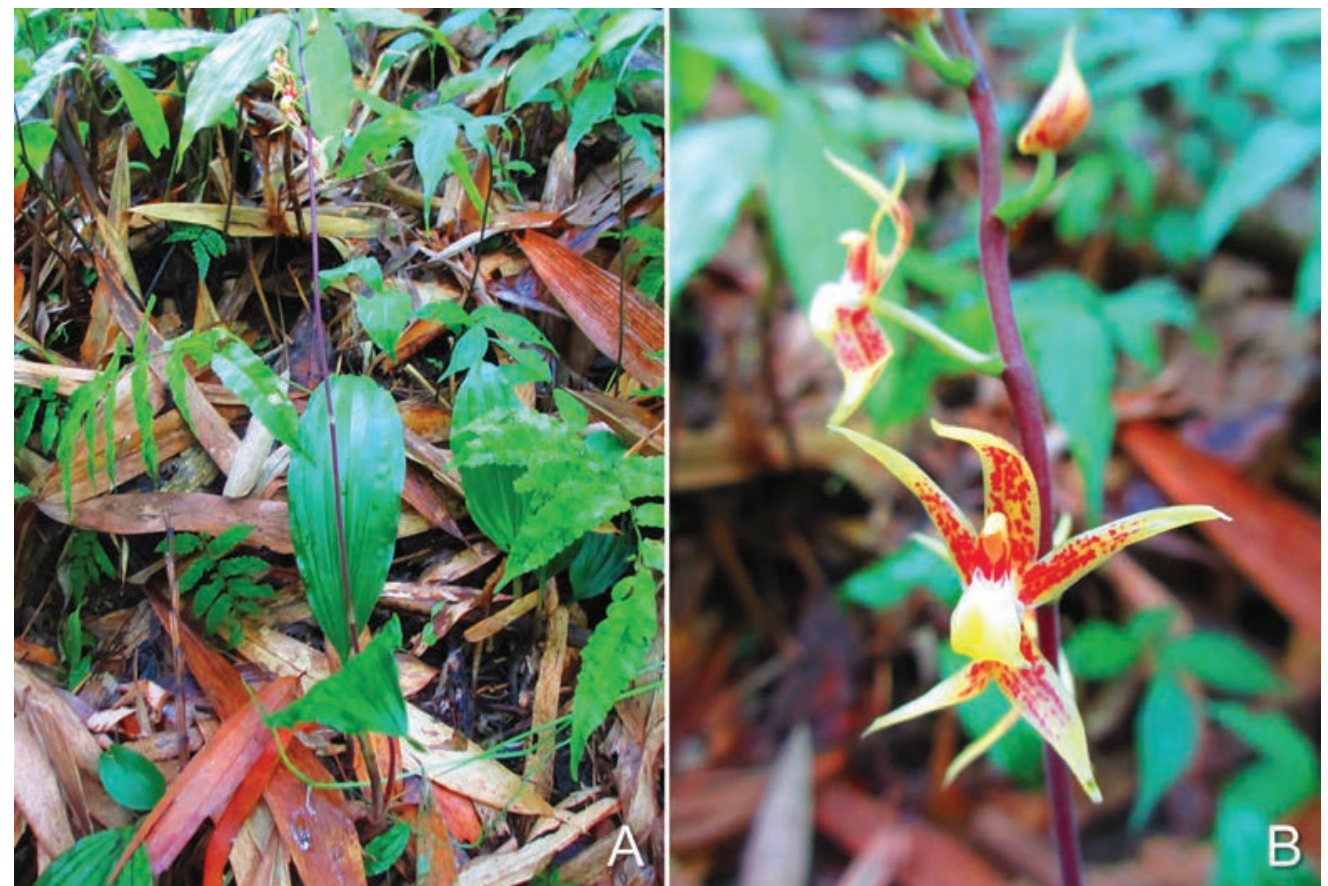

Fig. 2. Plocoglottis plicata (Roxb.) Ormerod. A. Habit. B. Detail of the flower. (Photos: Deby Arifiani) 
Styloglossum speciosum (Blume) T.Yukawa \& P.J.Cribb, Bull. Natl. Mus. Nat. Sci., Ser. B, 40(4): 150 (2014). - Calanthe speciosa (Blume) Lindl., Gen. Sp. Orchid. P1.: 250 (1833); Comber, Orchids Java: 93 (1990); Seidenfaden \& Wood, Orchids Penins. Malaysia Singapore: 175 (1992). - Amblyglottis speciosa Blume, Bijdr. Fl. Ned. Ind.: 371 (1825). - TYPE: Java, Blume s.n. (L, n.v.). (Fig. 4)

Pseudobulbs close together, with 4-7 leaves, hidden by leaf sheaths. Leaves 40-50 $\mathrm{cm}$ long, 8-10 cm wide, oblong, nerves prominent, acute, leaf sheaths overlapping. Inflorescences 45-80 cm long; sterile bracts 5, boat-shaped, 2-6 cm long; bracts caducous, absent during flowering. Flowers $3 \mathrm{~cm}$ wide, petals and midlobe of lip yellowish, sepals golden yellow; sepals and petals ovate, acuminate; lip three-lobed, with two basal rounded calli.

Distribution. Southeastern and southern China, Japan (Ryukyu), Taiwan, Vietnam, Thailand, Peninsular Malaysia, Sumatra, Java, Bali, Borneo, Sulawesi, Moluccas, Philippines.

Habitat. Primary forest.

Specimen examined. INDONESIA. Bali: Jembrana district: Mendoyo subdistrict, Batu Agung village, Panca Seming, Yeh Mesehe protected forest, $700 \mathrm{~m}$ asl., 17 May 2013, Y. Santika 542 (BO).

Vanilla albida Blume, Cat. Gew. Buitenz.: 100 (1823); Comber, Orchids Java: 76 (1990); Seidenfaden \& Wood, Orchids Penins. Malaysia Singapore: 127 (1992); Comber, Orchids Sumatra: 130 (2001). - TYPE: Java, Blume s.n. (holo L, n.v.).

Pseudobulbs absent. Stems climbing, with internodes 10-11 cm long, leaves alternate, aerial roots opposite leaves. Leaves lanceolate, 15-18 cm long, 3-4 cm wide, acute, petiole $1-1.5 \mathrm{~cm}$ long. Inflorescences axillary, bearing many flowers, floral bracts obtuse. Flowers open only on one day, at first widely open and then half-closing; sepals and petals pale yellow or yellowish green; lip white, but violet inside near the base, recurved, trumpet-like.

Distribution. Andaman Islands, Nicobar Islands, Sumatra, Java, Bali, Borneo.

Habitat. Lowland forest.

Specimen examined. INDONESIA. Bali: Jembrana district: Mendoyo subdistrict, Poh Santen village, Pasatan, forest surrounding Mt Mesehe, $360 \mathrm{~m}$ asl., 18 May 2013, D. Arifiani 1386 (BO). 


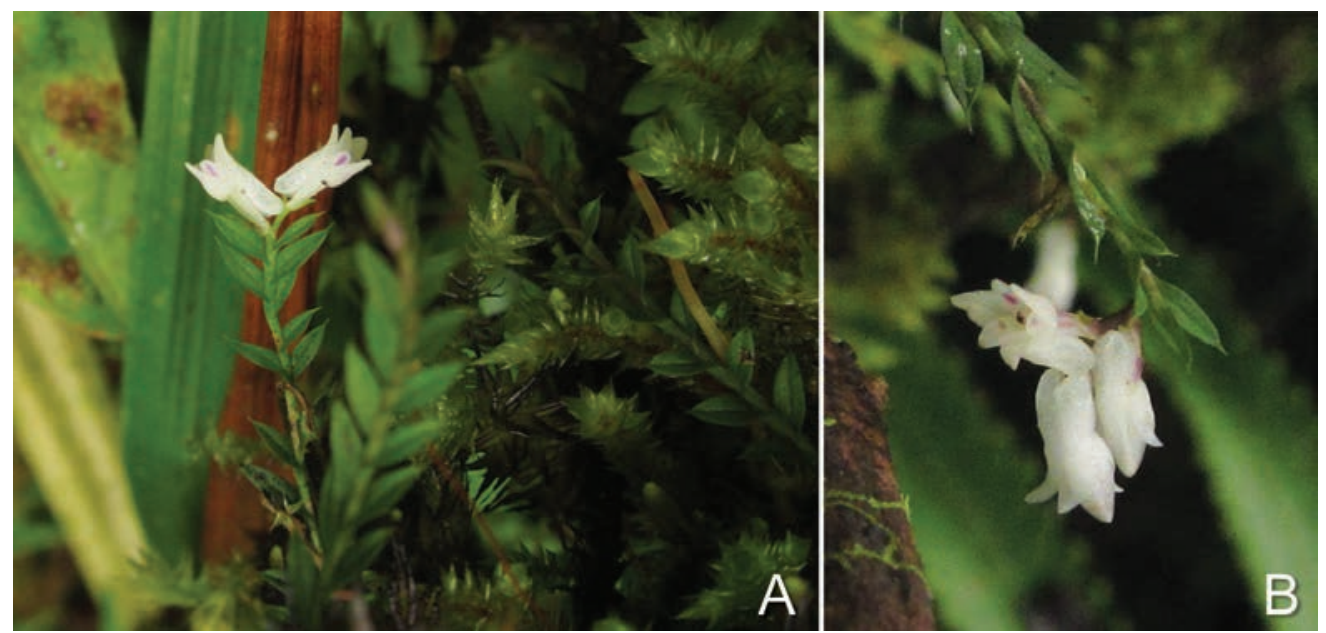

Fig. 3. Podochilus serpyllifolius (Blume) Lindl. A. Habit. B. Detail of the flower. (Photos: Yessi Santika)
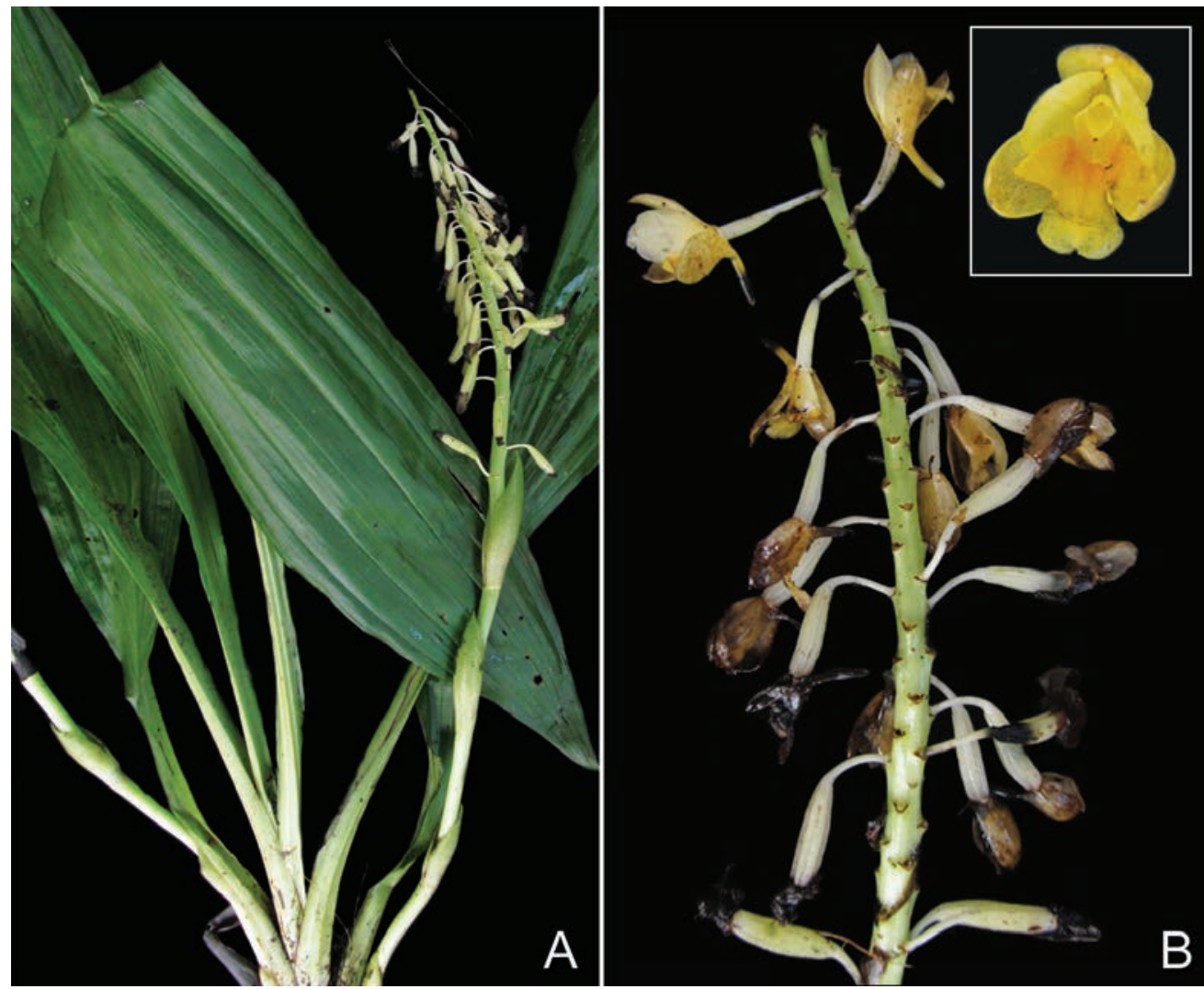

Fig. 4. Styloglossum speciosum (Blume) T.Yukawa \& P.J. Cribb. A. Habit. B. Detail of the inflorescence with flower in front view (inset C). (Photos: Yessi Santika) 
Table 1. Orchid species collected from Mt Mesehe and Mt Merbuk. (** = newly recorded species from Bali, names with cf. are based on sterile specimens).

\begin{tabular}{|c|c|c|}
\hline No. & Species name & Notes \\
\hline 1 & Appendicula cornuta Blume & $\begin{array}{l}\text { Mt Mesehe, moist forest, alt. 500-1500 m. } \\
\text { Epiphyte. }\end{array}$ \\
\hline 2 & Bulbophyllum lobbii Lindl. & $\begin{array}{l}\text { Mt Mesehe, moist forest, alt. 900-1000 m. } \\
\text { Epiphyte. }\end{array}$ \\
\hline 3 & Calanthe cf. masuca (D.Don) Lindl. & $\begin{array}{l}\text { Mt Merbuk, shady primary forest, alt. } 400- \\
1600 \mathrm{~m} \text {. Terrestrial. }\end{array}$ \\
\hline 4 & Calanthe flava (Blume) C.Morren & $\begin{array}{l}\text { Mt Merbuk, primary forest, alt. } 700-2200 \mathrm{~m} \text {. } \\
\text { Terrestrial. }\end{array}$ \\
\hline 5 & Calanthe zollingeri Rchb.f. ** & $\begin{array}{l}\text { Mt Mesehe and Mt Merbuk, lowland forest } \\
\text { along river, also in primary forest, alt. } 200- \\
550 \mathrm{~m} \text {. Terrestrial. }\end{array}$ \\
\hline 6 & Ceratostylis brevibrachiata J.J.Sm. & $\begin{array}{l}\text { Mt Mesehe, moist forest, alt. 800-1000 m. } \\
\text { Epiphyte. }\end{array}$ \\
\hline 7 & Dendrobium cf. montanum J.J.Sm. & $\begin{array}{l}\text { Mt Mesehe, moss forest, alt. 900-1000 m. } \\
\text { Epiphyte. }\end{array}$ \\
\hline 8 & Dendrobium plicatile Lindl. & $\begin{array}{l}\text { Mt Mesehe, lowland forest, alt. 500-800 m. } \\
\text { Epiphyte. }\end{array}$ \\
\hline 9 & Liparis condylobulbon Rchb.f. & $\begin{array}{l}\text { Mt Mesehe and Mt Merbuk, lowland forest } \\
\text { in open area, alt. } 500-800 \mathrm{~m} \text {. Epiphyte. }\end{array}$ \\
\hline 10 & Mycaranthes oblitterata Blume & $\begin{array}{l}\text { Mt Mesehe, lowland forest, alt. } 400-800 \mathrm{~m} \text {. } \\
\text { Epiphyte. }\end{array}$ \\
\hline 11 & Nervilia concolor (Blume) Schltr. & $\begin{array}{l}\text { Mt Merbuk, lowland forest in open area, alt. } \\
400-600 \mathrm{~m} \text {. Terrestrial. }\end{array}$ \\
\hline 12 & Phaius pauciflorus (Blume) Blume & $\begin{array}{l}\text { Mt Mesehe and Mt Merbuk, lowland forest } \\
\text { in open area, alt. } 400-700 \mathrm{~m} \text {. Terrestrial. }\end{array}$ \\
\hline 13 & $\begin{array}{l}\text { Pinalia cf. multiflora (Blume) } \\
\text { Kuntze }\end{array}$ & $\begin{array}{l}\text { Mt Mesehe, lowland forest in open area, alt. } \\
600-800 \text { m. Epiphyte. }\end{array}$ \\
\hline 14 & $\begin{array}{l}\text { Plocoglottis plicata (Roxb.) Ormerod } \\
* *\end{array}$ & $\begin{array}{l}\text { Mt Mesehe and Mt Merbuk, lowland forest } \\
\text { or primary forest, sometimes growing on } \\
\text { rocks, alt. 200-900 m. Terrestrial. }\end{array}$ \\
\hline 15 & $\begin{array}{l}\text { Podochilus serpyllifolius (Blume) } \\
\text { Lindl. ** }\end{array}$ & $\begin{array}{l}\text { Mt Mesehe, primary forest, alt. } 900-1000 \mathrm{~m} \text {. } \\
\text { Epiphyte. }\end{array}$ \\
\hline 16 & Spathoglottis plicata Blume & $\begin{array}{l}\text { Mt Merbuk, lowland forest in open area, alt. } \\
400-600 \mathrm{~m} \text {. Terrestrial. }\end{array}$ \\
\hline 17 & $\begin{array}{l}\text { Styloglossum speciosum (Blume) } \\
\text { T.Yukawa \& P.J.Cribb ** }\end{array}$ & $\begin{array}{l}\text { Mt Mesehe, primary forest, alt. } 700-850 \mathrm{~m} \text {. } \\
\text { Terrestrial. }\end{array}$ \\
\hline 18 & Trichotosia ferox Blume & $\begin{array}{l}\text { Mt Mesehe, primary moist forest, alt. } 800- \\
950 \text { m. Epiphyte. }\end{array}$ \\
\hline 19 & Tropidia angulosa (Lindl.) Blume & $\begin{array}{l}\text { Mt Merbuk, lowland forest, alt. 400-600 m. } \\
\text { Terrestrial. }\end{array}$ \\
\hline 20 & Vanilla albida Blume ** & $\begin{array}{l}\text { Mt Mesehe, lowland forest, alt. } 300-400 \mathrm{~m} \text {. } \\
\text { Terrestrial becoming climber. }\end{array}$ \\
\hline
\end{tabular}


ACKNOWLEDGEMENTS. This study was financially supported by the Research Center for Biology-Indonesian Institute of Sciences (DIPA 2013 and 2014). We are grateful to the Head of the Dinas Kehutanan Jembrana, to the staff for their valuable support, and to the people of Batu Agung village for their assistance during field work. Two anonymous reviewers are thanked for their valuable and constructive suggestions.

\section{References}

Anonymous (2014). Profil Kabupaten Jembrana 2014. 227 p. Bali: Badan Perencanaan dan Pembangunan Daerah dan Penanaman Modal, Pemerintah Kabupaten Jembrana, Bali.

Comber, J.B. (1990). Orchids of Java. UK: Royal Botanic Gardens, Kew.

Comber, J.B. (2001). Orchids of Sumatra. Kota Kinabalu: Natural History Publications (Borneo) \& UK: Royal Botanic Gardens, Kew.

eMonocot (2015). An online resource for monocot plants. http://e-monocot.org. (accessed on Jun. 2015).

Freudenstein, J.V. \& Chase, M.W. (2014). Phylogenetic relationships in Epidendroideae (Orchidaceae), one of the great flowering plant radiations: progressive specialization and diversification. Ann. Bot. 115(4): 665-681.

Girmansyah, D., Santika, Y., Retnowati, A., Wardani, W., Haerida, I., Widjaja, E.A \& Van Balgooy, M.M.J. (2013). Flora of Bali: An Annotated Checklist. Jakarta: Research Center for Biology-LIPI \& Yayasan Pustaka Obor Indonesia.

Rugayah, Retnowati, A., Windadri, F.I. \& Hidayat, A. (2004). Pengumpulan data taksonomi. In: Rugayah, E.A.Widjaja \& Praptiwi (eds) Pedoman Pengumpulan Data Keanekaragaman Flora. Pp. 5-42. Jakarta: Pusat Penelitian Biologi-Lembaga Ilmu Pengetahuan Indonesia.

Schuiteman, A., De Vogel, E.F., Vermeulen, J.J., Kessler, P. \& Vogel, A. (2014). Malesian Orchid Genera Illustrated. http://www.nationaalherbarium.nl/pubs/orchidweb/ Malesianorchidgenera.htm. (accessed on Oct. 2014).

Seidenfaden, G. \& Wood, J.J. (1992). The Orchids of Peninsular Malaysia and Singapore. Fredensborg: Olsen \& Olsen. UK: Royal Botanic Gardens, Kew \& Singapore: Singapore Botanic Gardens.

Stevens, P.F. (2015). Angiosperm Phylogeny Website, version 12, July 2012 [and more or less continuously updated since]. http://www.mobot.org/MOBOT/research/APweb/ (accessed on Jul. 2015).

Van Balgooy, M.M.J. (1987). Collecting. In: De Vogel, E.F. (ed) Manual of Herbarium Taxonomy, Theory and Practice. Pp. 14-19. Jakarta: UNESCO.

Wood, J.J., Beaman, R.S. \& Beaman, J.H. (1993). The Plants of Mount Kinabalu, 2. Orchids. UK: Royal Botanic Gardens, Kew. 
